\title{
Proliferation and Adhesion of Endothelial Cells on the Surface of Titanium Implant
}

\author{
Jing-Ying ZHANG ${ }^{1, a,{ }^{*}}$ \\ ${ }^{1}$ Medicine School, Dalian University, Dalian 116622, China \\ ajingyingzhang2014@foxmail.com \\ ${ }^{*}$ Corresponding Author
}

Keywords: Endothelial Cells, Dental Implants, Proliferation, Adhesion.

\begin{abstract}
To observe the proliferation and adhesion of umbilical cord endothelial cells (HUVEC) on the titanium (Ti) surface with large particles blasted-etched (SLA) treatments and further clarify the mechanism of action of each other between the Ti implant surface and the body. Methods: The human umbilical cord endothelial cells are cultured on the surface of SLA-Ti disks and culture plates. The proliferation of endothelial cells is detected by MTT method and adhesion of endothelial cells observed by PI fluorescence staining and western-bolt detection of protein of VE-cadherin. Results: The proliferation of endothelial cells on the titanium surface is better than the culture plate surfaces, the adhesion cells on titanium surface are more numbers, cell morphology is more extended, titanium surface expression of VE-cadherin protein higher than the culture plate surface. Conclusion: The SLA Ti surface can promote the proliferation and adhesion of endothelial cell and could promote the early healing process of osseointegration.
\end{abstract}

\section{Introduction}

Dental implant dentures have become the best way to repair edentulous dentition defect. Titanium implants have been used in clinical for decades and have achieved a high success rate due to its excellent mechanical properties, corrosion resistance and biocompatibility (20-month cumulative survival rate was 94.6\%, 7-year survival rate 90.6\%)1. Aseptic loosening of titanium implant cases occurred at intervals and the mechanism is not very clear, which are located in the interface of implants and body and caused by the wound healing process.

After implantation into the body of titanium implants, new blood vessels begin to form at the wound site. The blood vessels formation, beginning from endothelial cells, is major process determine planting success 2 . Endothelial cell activation surrounding tissue wound healing response, the inflammatory phase of wound healing at the implantation site of vascular damage, blood flow begins to solidify and form a fibrin clot, while the activation of pro-inflammatory leukocytes such as neutrophils and monocytes cell aggregation in the wound area, leukocyte phagocytosis and by generating ROS remove cell debris and metal particles produced by corrosion3. The leukocytes from the blood to reach the wound site through the vessel wall, the production of cytokines (e.g. TNF- $\alpha$ ), recruit more leukocytes and activation endothelial cells, stimulation of endothelial cells upregulate the expression of adhesion molecules; and binds to E-selectin surface of endothelial cells, regulating cell adhesion processes (e.g., by expression of ICAM-1); activate endothelial cells to adjust its skeletal structure, allowing leukocytes through endothelial cell between contacts (e.g. by CD31 regulation)4. In addition, activated endothelial cells release pro-inflammatory cytokines (such as E-selectin, ICAM-1 and IL-8), attracted more leukocyte activation and expand the inflammatory response. Around the implant can be observed in a transient inflammatory cytokines (such as IL-1 $\beta$, IL-6 and TNF- $\alpha$ ) expression. Endothelial cells in the wound healing process involved in the formation of early and late tissue inflammation in the proliferative phase of wound healing, fibroblasts and endothelial cells to form new blood vessels; in situ around the reconstruction of implant mineralized tissue formation, maintaining long-term implant osseointegration5. Based on the clinical use up large particles blasted - processing of titanium etching (SLA-Ti) surface of cultured endothelial cells, biological response study implant surface of endothelial cells, the 
mechanism described implant surface of endothelial cell responses. In this study, we compare the proliferation and adhesion of umbilical cord endothelial cells on the titanium (Ti) surface with large particles blasted-etched (SLA) treatments and further clarify the mechanism of action of each other between the titanium implant surface and the body.

\section{Materials and Methods}

\section{Cell Culture}

Human umbilical vein endothelial cells were from the Shanghai Sinovac Biotech Ltd. and cultivated in 1640 medium (Hyclone) supplemented with 10\% fetal bovion serum (Hyclone), $1 \%$ penicillin/streptomycin (Hyclone) in a humidified atmosphere at 5\% $\mathrm{CO} 2$ air atmosphere at $37{ }^{\circ} \mathrm{C}$. The medium was changed every three days, cells were subcultured after grown to $80 \%$ confluence. The endothelial cells in passage four sere seeded on Ti disks treated with sandblasting and acid etching (SLA) with or 24-well culture plates.

\section{Cell proliferation}

Ti disks (18 samples) were placed into 3 sterile 24 -well reaction plates. Endothelial cells $(500 \mu 1$, $1 \times 105$ cells $/ \mathrm{ml}$ ) were aliquoted into the three sterile 24 -well plates containing the samples and placed in a $37{ }^{\circ} \mathrm{C}, 5 \% \mathrm{CO} 2$ intracellular incubator with saturated humidity for 1,2 and 3 days. At the corresponding time points, the samples were carefully transferred to unused 24-well plates and then rinsed with PBS. Subsequently, the samples were cultured with $30 \mu \mathrm{M}$ of $5 \mathrm{mg} / \mathrm{ml}$ thiazolyl blue (MTT) per well, for $4 \mathrm{~h}$ under the same conditions, and the MTT then aspirated. After washing with PBS, $700 \mu$ of dimethyl sulfoxide (DMSO) per well was added and the solution agitated for $10 \mathrm{~min}$. The liquid in each well of 24 -well plates was divided into four parts $(150 \mu \mathrm{l})$ and transferred to a 96-well plate to determine the absorbance value at $490 \mathrm{~nm}$ using a microplate reader (Thermo Fisher, USA).The proliferation of HUVEC on the surfaces of Ti disks or culture plates incubation were protected from light, reader OD value in the detection wavelength of $450 \mathrm{~nm}$ conditions.

\section{Cell Adhesion}

Ti disks were placed individually, in quadruplicate, into the sterile 24-well plates and an endothelial cells suspension at a density of $5 \times 104 \mathrm{cells} / \mathrm{ml}(100 \mu \mathrm{l})$ was pipetted onto the surface of each sample. After 4 h, $500 \mu \mathrm{l}$ complete medium was added to each well and cells cultured for 48 hours. The samples were rinsed three times with PBS following the removal of the medium. Samples in each well were cultured with $500 \mu \mathrm{l}$ coloring agent $(0.5 \mathrm{mmol} / \mathrm{ml} \mathrm{PI})$ at $37{ }^{\circ} \mathrm{C}$ for 15 min and observed under a fluorescence microscope (OLYMPUS IX71 / DP70).

\section{Western Blot}

After 3 days on incubation, endothelial cells were lysed in RIPA lysis buffer. After-wards, the lysate was cleared by centrifugation $(12,000 \mathrm{rpm})$ at $4{ }^{\circ} \mathrm{C}$ for $15 \mathrm{~min}$. The protein was quantified and separated by $10 \%$ SDS-PAGE gel and then transferred to PVDF membranes. After blocked with $5 \%$ non-fat milk for $60 \mathrm{~min}$ at room tempereatre, the membranes were incubated overnight at $4{ }^{\circ} \mathrm{C}$ with primary monoclonal antibodies against VE-cadherin $(1: 1000)$ and $\beta$-actin( cell signaling, at a 1:300 dilution), followed by horseradish peroxidase conjugated secondary antibody(at a 1:300 dilution) for $120 \mathrm{~min}$ at room temperature. The immunoreative bands were visualized using a chemiluminescenc kit. The intensity of the protein bands was quantified by densitometric scanning using image software and normalized to $\beta$-actin.

\section{Statistical Analysis}

For cell culture studies, datum is presented from one of three separate experiments, all of which yield comparable result. For any given experiment, each value represents the mean plus or minus the standard error of the mean of six individual cultures (mean \pm S.D.). The datum is analyzed by an analysis of variance, and the statistical significance was determined with Bonferroni's modification 
of the student $\mathrm{t}$ test or one way ANOVA. P values less than or equal to 0.05 were considered significant.

\section{Results and Discussion}

\section{Culture of Endothelial Cells}

After the cells were subcultured three generations, the cells were fully spread with varied shapes as triangular, fusiform and irregular polygonal shape, which were shown in Fig. 1. Light microscopy showed the nucleus and abundant cytoplasm. The cells were growth in colonies and tightly packed. With time culture, cell extended many a protrusion extended by mutual contact between the cell processes, integrated into the film. Subculture about 10 days, the cells almost covered with flat, form a monolayer cells, cell clusters fusion.

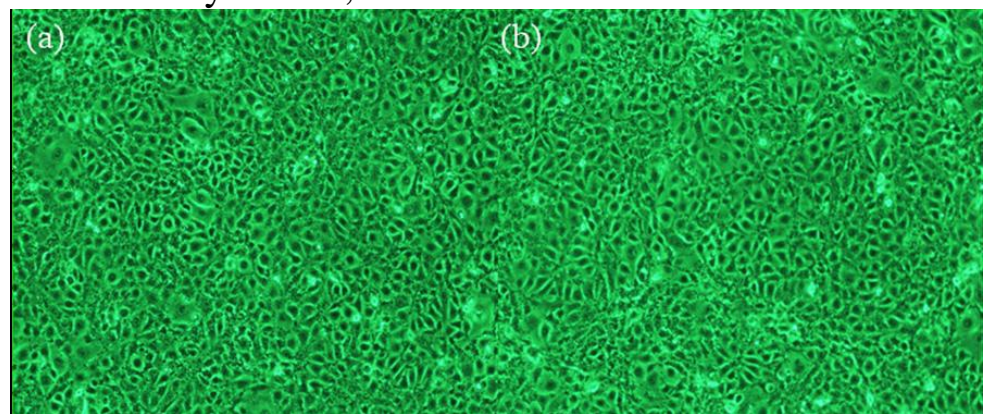

Fig. 1 Primary and Passage Culture of HUVECs of 3d (P3) Scale Bar: $100 \mu \mathrm{m}$

\section{Proliferation of Endothelial Cells}

The proliferated of endothelial cells on the surfaces of $\mathrm{Ti}$ and culture plates was determined by MTT assay. The number of HUVECs growing on the Ti surface significantly differed from that found growing on the culture plate surfaces $(\mathrm{P}<0.05$, Fig. 2$)$. With the extension of culture time, OD values of the experimental and control groups increase, on the $3 \mathrm{~d}$ presents the highest. These findings suggest that Ti treated with SLA can enhance HUVEC growth on the surface of Ti and moreover, Ti treated with SLA is superior to culture plates for the promoting growth of these cells.

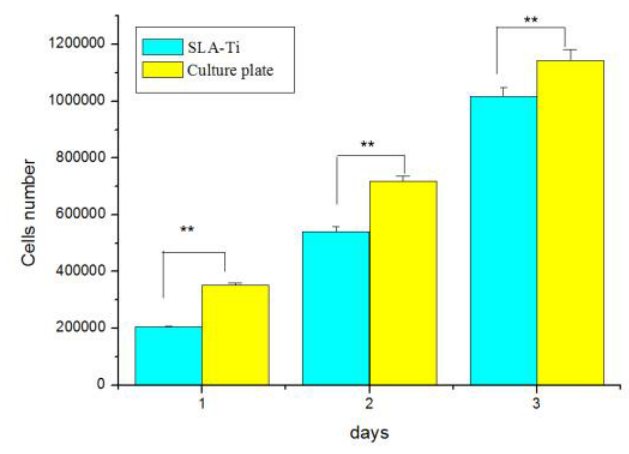

Fig. 2 Proliferation of HUVEC on the Surface of Ti and Culture Plate

\section{Adhesion of Endothelial Cells}

Early cell adhesion is a biological signal to initiate a series of cellular behaviors and includes four successive overlapping phases: cell attachment, cell spreading, actin cytoskeleton formation and focal adhesion formation6. Endothelial cells can proliferate, express genes, differentiate, produce apoptosis, migrate and even play a function on the sample surface. The long-term biological response is largely dependent on cell adhesion, which is the key to the success of implantation7. After SLA treatment, PI-labeled endothelial cells on the Ti surface proliferated and became more extended in shape, changing from spherical or triangular, to polygonal or fusiformal (Fig. 3). Thus, 
the SLA-treated sample surface exhibited the porous structure and increased roughness that could promote the growth and proliferation of HUVECs on the sample surface. Therefore, the adhesion ability of endothelial cells was enhanced. Compared with other surface modification methods, this method is relatively simple and does not require high temperature and high pressure conditions, which is suitable for implants with complex surface morphology. After cultured on the surface of the sample, the cells presented characteristic morphology, under the high-magnification, the cell filopodia attach to the materials tightly, the growth of endothelial cells on the metals is in good condition. With prolonged incubation, the cell density increases. There is no difference on the number and morphology between the experimental group and the control group.

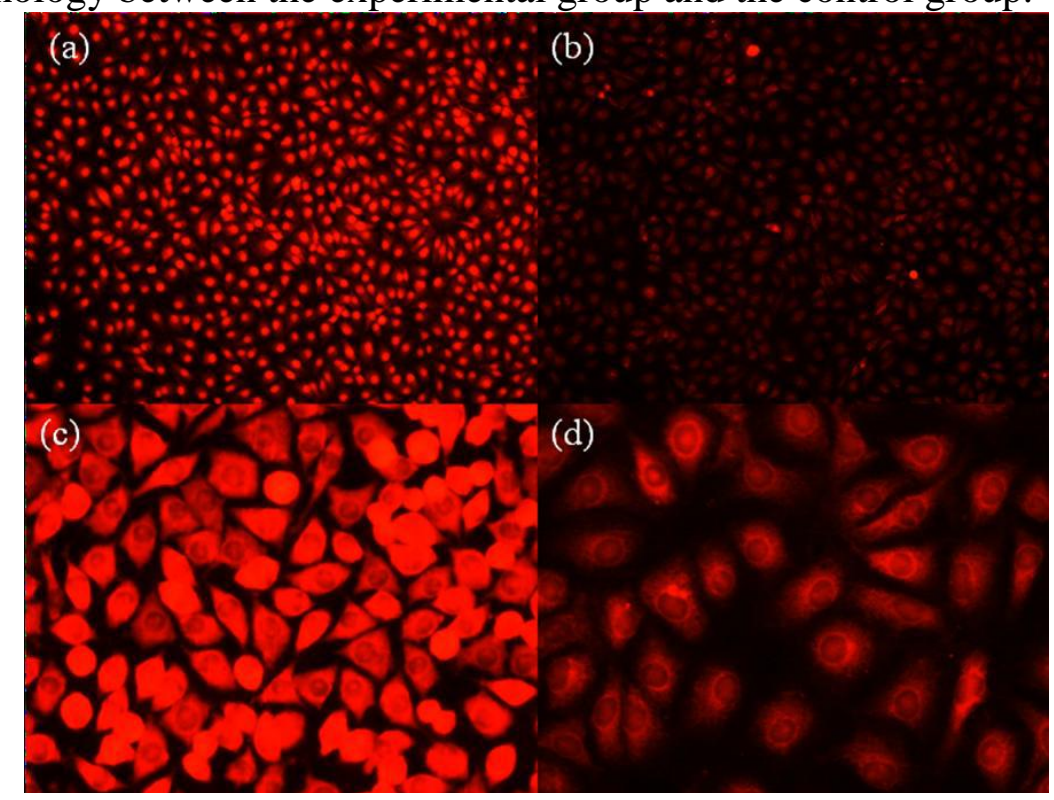

Fig. 3 Fluoresein-micrscope Images of Attached HUVECs Cultured for 48h on the Sample Surface:

(a) (c) SLA treated Ti, (b) (d) culture plate surface, scale bar: (a) (b) $400 \mu \mathrm{m}$, (c) (d) $100 \mu \mathrm{m}$

\section{Western Blot}

The expressions of VE-cadherin on the surface of Ti present insignificant difference ( $\mathrm{p}>0.05)$. The clearest sign of cell adhesion function of cell adhesion molecule expression, and for implant materials, requires not only material capable of promoting cell growth, cell and requires a complete biological function. Cell adhesion molecules are a large class of molecules collectively interconnections between cells and cell-mediated or cell and matrix, comprising: a cadherin, selectin, integrin family, etc., involved in cell signaling, growth and differentiation and other physiological process8. Cadherin is a kind of calcium-dependent cell adhesion molecule, is a kind of single-chain transmembrane glycoprotein. Vascular endothelial cadherin (VE-Cadherin), one of the most specific molecular endothelial cells, is a major endothelial cell adhesion molecule-Fi, for the maintenance of endothelial cell polarity and integrity are essential to maintain stable blood vessels, regulation of vascular permeability9

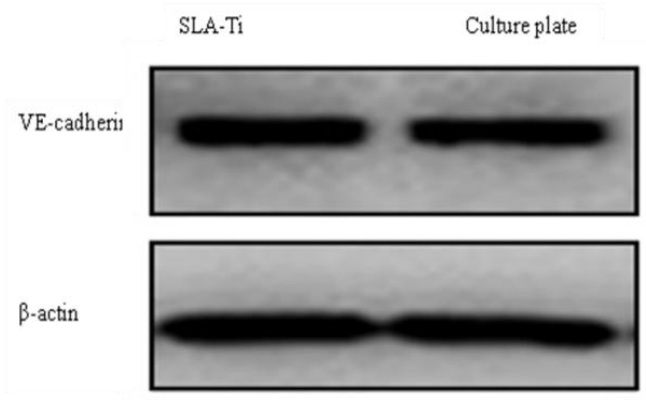

Fig.4 The relative expression of VE-Cadherin after incubation for $3 \mathrm{~d}$ with specimens 


\section{Conclusion}

In this study the proliferation of endothelial cells on the titanium surface is better than the culture plate surfaces, the adhesion cells on titanium surface are more numbers, cell morphology is more extended, titanium surface expression of VE-cadherin protein higher than the culture plate surface. The SLA-Ti surface can promote the proliferation and adhesion of endothelial cell and could promote the early healing process of osseointegration. Therefore, SLA-treated Ti has the good biological activity of endothelial cells.

\section{Acknowledgements}

This research was supported by the Liaoning Education Bureau (grant code 2013481), the Science and Technology Plan of Dalian City (grant code 2013E11SF057), China Postdoctoral Fund (grant code 2014M551097), Postdoctoral Initial Fund of Liaoning Province (grant code 20141198). The authors would like to acknowledge funding from SUNTEC Titanium Ltd. For the purchase of Ti and $\mathrm{Ti}$ alloys.

\section{References}

[1] Katyayan PA, Katyayan M, Shah RJ. Rehabilitative considerations for dental implants in the diabetic patient. Journal of Indian Prosthodontic Society. Sep 2013; 13(3): 175-183.

[2] Unger RE, Peters K, Sartoris A, Freese C, Kirkpatrick CJ. Human endothelial cell-based assay for endotoxin as sensitive as the conventional Limulus Amebocyte Lysate assay. Biomaterials. Mar 2014; 35(10): 3180-3187.

[3] Tsaryk R, Kalbacova M, Hempel U, et al. Response of human endothelial cells to oxidative stress on Ti6Al4V alloy. Biomaterials. Feb 2007; 28(5): 806-813.

[4] Nourshargh S, Alon R. Leukocyte Migration into Inflamed Tissues. Immunity. Nov 20 2014; 41(5): 694-707.

[5] Tsaryk R, Peters K, Barth S, Unger RE, Scharnweber D, Kirkpatrick CJ. The role of oxidative stress in pro-inflammatory activation of human endothelial cells on Ti6Al4V alloy. Biomaterials. Nov 2013;34(33):8075-8085.

[6] DeMali KA, Sun X, Bui GA. Force transmission at cell-cell and cell-matrix adhesions. Biochemistry. Dec 16 2014; 53(49): 7706-7717.

[7] Deng Z, Yin B, Li W, et al. Surface characteristics of and in vitro behavior of osteoblast-like cells on titanium with nanotopography prepared by high-energy shot peening. International journal of nanomedicine. 2014; 9: 5565-5573.

[8] Bhella D. The role of cellular adhesion molecules in virus attachment and entry. Philosophical transactions of the Royal Society of London. Series B, Biological sciences. Feb 5 2015; 370(1661).

[9] Gavard J. Endothelial permeability and VE-cadherin: a wacky comradeship. Cell Adh Migr. 2014; 8(2): 158-164. 